\title{
The Phenomenon of Pain
}

The Phenomenon of Pain

Serge Marchand $\mathrm{PhD}$

ISBN: 978-0-931092-91-6

(C) 2012 IASP Press

Price: US \$70.00 (IASP Members \$55.00)

$\mathrm{T}$ his book is written by Dr Serge Marchand, a highly respected neurophysiologist, professor of neurosurgery and director of the Etienne-Le Bel Research Center of Sherbrooke University Hospital, Sherbrooke, Quebec. He is a world-recognized expert in the field of pain mechanisms and treatment.

The introduction and the first chapter, 'What is Pain?' provide a good general overview of the various issues with regard to pain. It includes John Loeser's useful model of four pain components (nociception, pain, suffering and pain behaviour - to which one might have added social determinants), with some suggestions for modification, as well as a comprehensive taxonomy of various pain terms and definitions that are based on the International Association for the Study of Pain taxonomy. It is a very good chapter that sets the stage for what follows.

Chapter 2, 'Concepts of Neurophysiology', is written in a conversational style that is easy to read and has clear diagrams. It is logically organized from the cellular level to the brain and contains much useful information, providing a basis for the following chapter on the neurophysiology of pain. It is a good source of basic information for the medical student and generalist reader.

Chapter 3, 'Neurophysiology of Pain', is a comprehensive chapter that benefits the general reader but also those involved in specialized fields of pain who want to obtain succinct clarification regarding some aspect of the neurophysiology of pain. It moves in a logical fashion from the peripheral nervous system to the central nervous system. I enjoyed the sections on referred pain from a clinical point of view, and also the review on temporal and spatial summation, and the account of primary and secondary hyperalgesia. There is a good account of the endogenous mechanisms of pain modulation and, particularly, descending mechanisms, in which the author has particular interest and expertise. It was nice to see here and elsewhere in the book the important early contributions of DV Reynolds mentioned, which often seem neglected in this area. The clarification of sometimes misunderstood concepts, such as diffuse noxious inhibitory controls (DNIC) of wind-up and central sensitization, are clearly elucidated.

Chapter 4, 'Theories of Pain' succinctly outlines the details and evolution of pain theories including specificity, pattern and gate control. It emphasizes the importance of the contributions of each because the older theories tend to be neglected somewhat. The seminal contributions of Ronald Melzack and Patrick Wall have appropriate prominence. The author's contribution of the circular pain model, regarding the interaction of the various components of the pain experience, is innovative and useful. The emphasis on the modest results of electrical stimulation, including transcutaneous electrical nerve stimulation (TENS) and deep brain stimulation, are important to review. Overall, this is a readable chapter that contains information for the general reader, the student with an increasing interest in pain, and the clinician wishing for a good review of these theories.

Chapter 5 is a comprehensive review of pain measurement scales for the verbal and nonverbal patient at all ages. The student and experienced clinician will benefit here both from the detail and for references for further reading. The beginner student can use this chapter as a springboard for developing his or her individual pragmatic approach for their own context for diagnosis and ongoing assessments. Of particular importance is the emphasis on the credibility of the patient's report, the differences between threshold and tolerance, and the importance of considering the multidimensional aspect of the pain experience.

Chapter 6, 'Pharmacological and Surgical Approaches to Pain Control', is an overview that includes references for further reading. It covers the main categories of drugs used for pain relief as well as a review of ablative and neuromodulation-type surgical procedures. This chapter voices a balanced view of opioids in the current climate of opioidphobia, commenting that the risks are small when they are used carefully and that they are necessary for some patients. The section on anticonvulsants was of interest because the author rightly discusses their effect on sodium channels, but points out that many of these drugs also act on calcium channels, gamma-aminobutyric acid mechanisms and $\mathrm{N}$-methyl-D-aspartate receptors. The important point here is that most of the drugs used are not 'magic bullets' or mechanismbased, but rather relatively 'dirty drugs' that act on multiple mechanisms. This may, in fact, be important because monotherapy so often fails and the use of combinations of drugs that act in different ways on excitation and inhibition is often necessary to obtain optimal relief. The overview of cannabinoids presents the opinion that they were of modest effect in clinical trials. The cautious view is voiced that ablative surgery and neurostimulation are limited in their application and only appropriate for refractory patients.

Chapter 7, 'Non-Pharmacological Pain Treatments', divides these into three neurophysiological models: those increasing inhibition, such as conventional TENS; those involving painful stimuli and involving DNIC; and control from higher centers such as cognitive behavioural approaches, relaxation, hypnosis and placebo. A detailed discussion of TENS follows that provides useful suggestions to maximize this modality including recommendations regarding frequency, duration, wavelength, localization and dietary manipulation, particularly caffeine. As the author states, it is important to remember that TENS produces mild analgesia, generally reducing pain by less than $50 \%$; thus, maximizing its effect is critical. Pain modulation by DNIC involving pain to relieve pain includes some types of acupuncture, vigorous massage and a form of TENS, and is a very good discussion of an often-misunderstood mechanism. It includes recommendations for use, again differentiating acupuncture-like TENS from conventional TENS and making recommendations regarding the parameters mentioned before. The section on control by higher centres includes discussions on the placebo effect, relaxation therapy, hypnosis and biofeedback, all important modalities for multidisciplinary and interprofessional approaches. I particularly enjoyed the discussion on the placebo effect, which emphazises that often the perception of this is

This review was published previously in the Journal of Pain and Symptom Management 
pejorative but that this may be one of the most important modalities that we have at our disposal. Some elaboration on the social determinants of pain would have been of interest. Relaxation therapy, biofeedback and hypnosis are also important modalities and are comprehensively discussed here. The conclusions of this section are that the analgesia produced by these different approaches is not negligible, and the advantages are that they make the patient independent and have virtually no side effects. The author cautions that the effect of any one modality is most likely modest, that it is important for caregivers to adopt a critical position based on scientific evidence to distinguish a valid treatment from a treatment built on charlatanism, and that these modalities are very easily subject to abuse.

Chapters 8, 'Pain and Age', covers the range of ages from newborn to children to the elderly. It describes important research regarding the maturation of nociceptive pathways in pain modulation systems, the common types of pain observed in children, and treatment options, both pharmacological and nonpharmacological, as well as pain in the elderly. Different outcome measures are reviewed that are necessary for nonverbal patients at the extremes of age, who present a particular challenge for those caring for these individuals.

Chapter 9, 'The Differences Between Men and Woman and Pain: More Than a Matter of Perception', nicely reviews the neurophysiological differences between sexes including genetic, psychosocial factors, mood disorders, cognitive factors and stereotypes.

Chapter 10, 'Pain: From Innate to Acquired', is a short chapter dealing with the role of innate factors such as genetics and sex. It uses codeine as a pertinent example, discussing genetic differences that lead to differences in codeine metabolism to morphine and result in ultra- and poor metabolizers, the variability of analgesia that this causes and the risk this poses for overdose among individuals such as ultrametabolizer Africans. The chapter goes on to discuss other innate influences, such as endogenous pain control mechanisms, and subsequently clarifies the terms race, culture, and ethnicity and the role of culture. The final conclusion, as in the rest of this book, is that although these issues are important to consider, the patient is front and foremost in providing an account of their pain and that we should not be biased by some of these factors.

Chapter 11, 'Pain Clinics', builds on the book's content to this point that pain is a complex phenomenon and many, if not most, patients will not be adequately treated with a unidisciplinary approach. The chapter also emphasizes the difficulty in treating many chronic noncancer pain patients. It begins with an important distinction - ie, the distinction between multidisciplinary and interprofessional pain clinics. Currently, there appears to be a misconception about these terms. The focus, then, is on the true interprofessional team model and the components of the team and, importantly, the selection of appropriate patients for an intensive interprofessional approach that would include consideration of age, sex, level of education, intensity of pain, number of surgeries, number of years since pain onset, site of pain, use of pain medications, compensation, obesity and smoking as being some factors to consider. Appropriate references are given to the International Association for the Study of Pain's criteria and for accreditation requirements for a true interprofessional pain team. This is of interest to those considering forming such a team and also in the evaluation of a current team approach.

The conclusion of the book is that pain is a complex phenomenon, and the contents of the book certainly reflect this statement. The necessity of understanding the neurophysiology and psychology of pain is emphasized. The author comments that it is unlikely that there will be a 'magic bullet' or a 'miracle pill' to solve all the problems of pain. He emphasizes the need for an interprofessional approach and points out that there is a basic knowledge that different professions can share to secure good communication and collaboration. Complex therapeutic strategies are often required. The importance of a skilled caregiver in helping the patient make informed decisions is discussed. The author emphasizes that pain is a subjective experience, is personal and is communicated differently based on sex, age, culture and experience. The patient should be the main collaborator on the health care team. There is an emphasis on pain being dynamic and the necessity for follow-up over time, with repeated measurements of pain and the effects of treatment as well as the monitoring of sleep, mood and function. A final cautionary note is made that "the patient may become an easy victim for those who practice questionable approaches". In this area, the knowledgeable caregiver's advice will be essential to assist the patient in making informed choices. The book finishes with a comprehensive glossary of pain terms and a useful index.

In summary, I was pleased to have the opportunity to review this excellent book, which is a useful resource on pain for this readership, both for the beginner student and also the seasoned clinician seeking succinct and current information. The research data provided are, in this reviewer's opinion, informed by the wisdom of clinical experience. It is also suitable for the patient and their caregiver who seek more information, in that the writing style is clear and it is very readable.

C Peter N Watson MD FRCPC
University of Toronto
Toronto, Ontario 


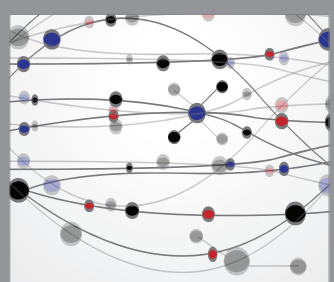

The Scientific World Journal
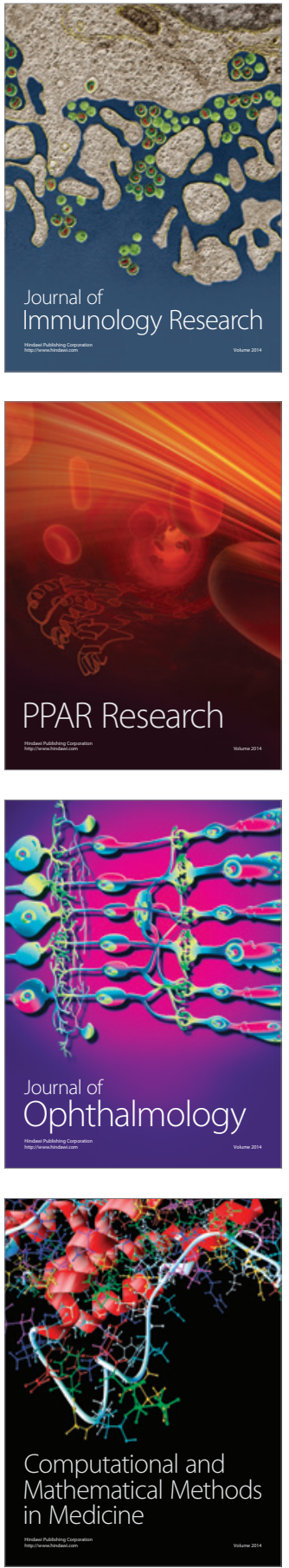

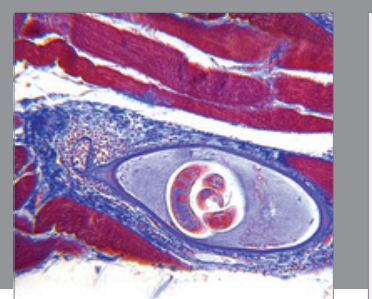

Gastroenterology Research and Practice

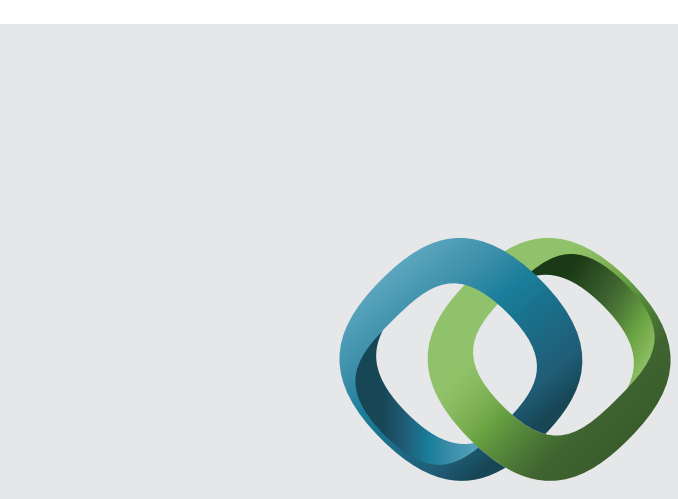

\section{Hindawi}

Submit your manuscripts at

http://www.hindawi.com
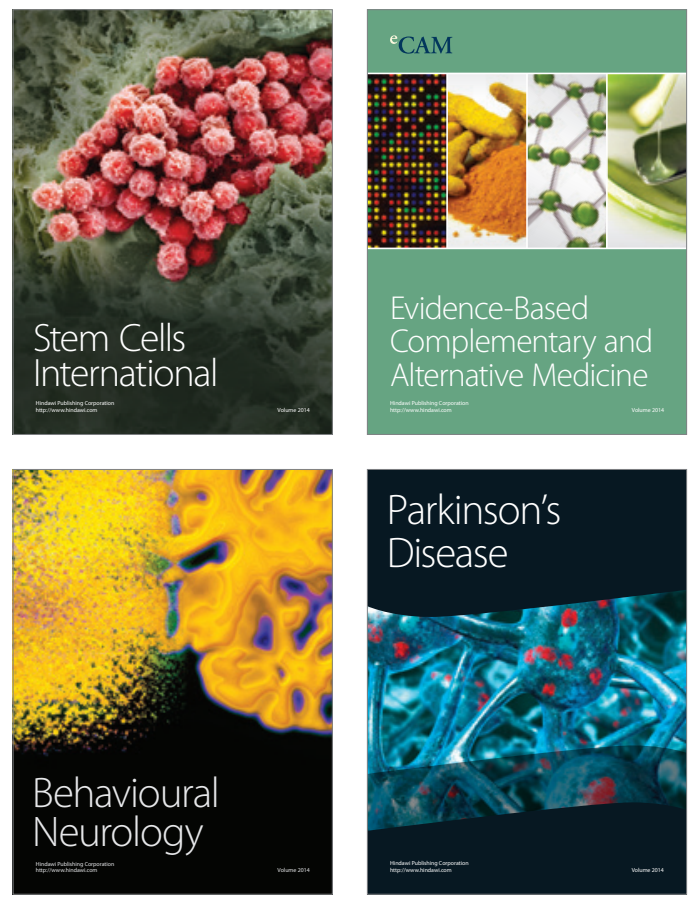
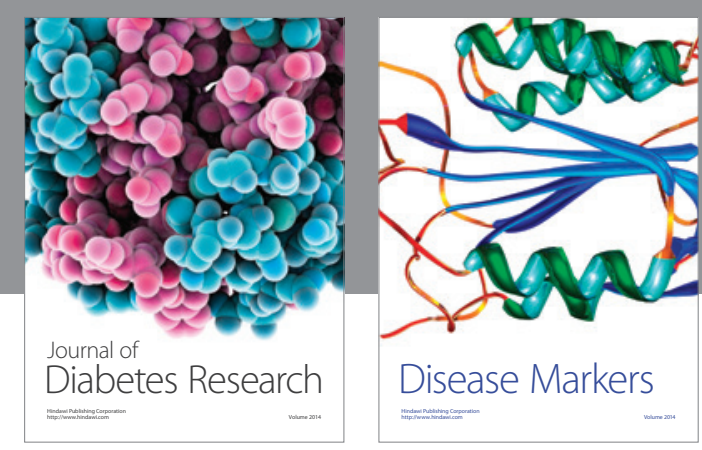

Disease Markers
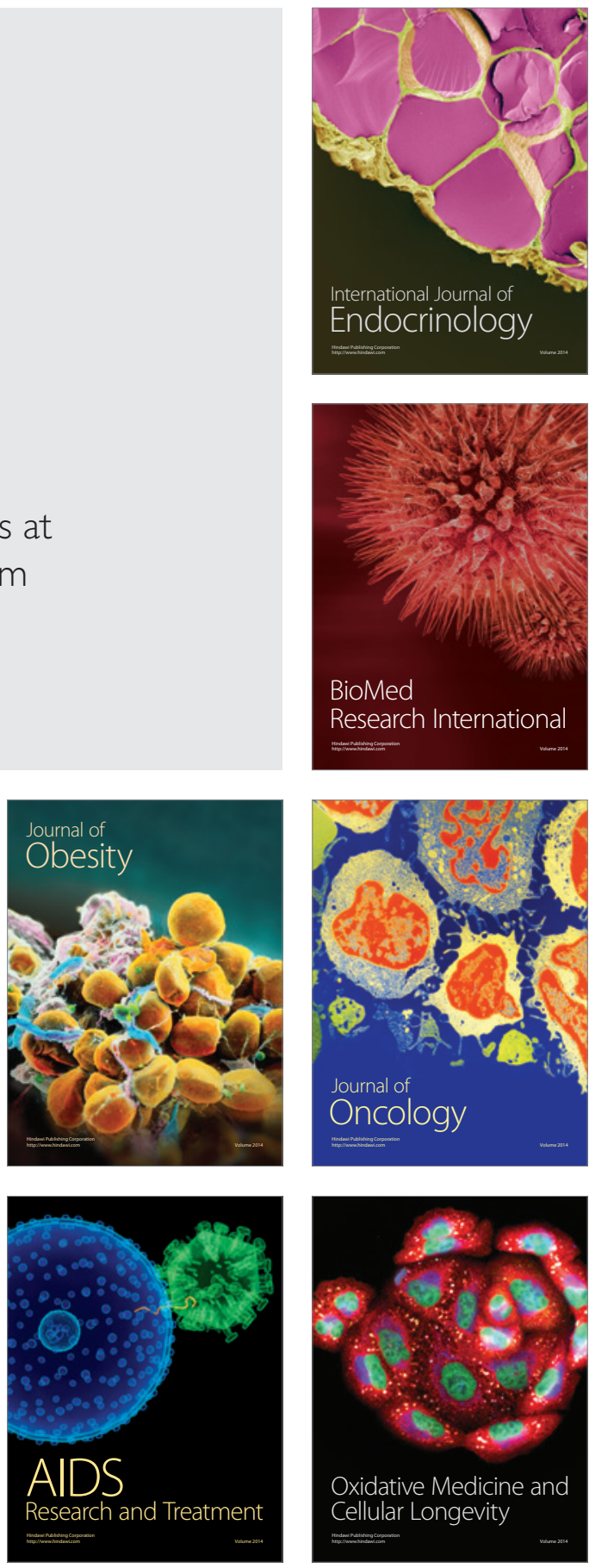